\title{
Nouvelles visibilités de la religion dans l'arène du développement
}

L'implication des élites chrétiennes et musulmanes dans les politiques publiques en Afrique

Mayke Kaag et Maud Saint-Lary

\section{(2) OpenEdition}

Journals

Édition électronique

URL : http://journals.openedition.org/apad/4073

DOI : 10.4000 /apad.4073

ISSN : 1950-6929

Éditeur

LIT Verlag

Édition imprimée

Date de publication : 1 août 2011

Référence électronique

Mayke Kaag et Maud Saint-Lary, « Nouvelles visibilités de la religion dans l'arène du développement », Bulletin de l'APAD [En ligne], 33 | 2011, mis en ligne le 19 mars 2012, consulté le 07 septembre 2020. URL : http://journals.openedition.org/apad/4073; DOI : https://doi.org/10.4000/apad.4073

Ce document a été généré automatiquement le 7 septembre 2020

Bulletin de l'APAD 


\title{
Nouvelles visibilités de la religion dans l'arène du développement
}

L'implication des élites chrétiennes et musulmanes dans les politiques publiques en Afrique

\author{
Mayke Kaag et Maud Saint-Lary
}

\section{Introduction}

1 En dépit d'une vision couramment répandue qui relègue le religieux au rang des forces rétrogrades, en Afrique les idées et les acteurs religieux ont toujours constitué des vecteurs $\mathrm{du}$ changement social et ont été importants pour les initiatives de développement. L'engagement des missions catholiques ou des élites islamiques dans le domaine de l'éducation à l'époque coloniale en témoigne (Cooper 2006 ; Brenner 2001). Toutefois, à partir des années 1950-1960, alors que les théories de la modernisation et leurs défenseurs se sont mis à dominer la scène, ces engagements des religieux dans les affaires publiques, semblent, pour leur part, s'en être éclipsé. Ce n'est que très récemment que la religion a été redécouverte comme force de changement (Ter Haar \& Ellis 2006). Il faut dire qu'en trente ans, une double mutation s'est opérée, tant dans le monde du développement que du point de vue du paysage religieux, lui-même en plein renouvellement.

\section{Le tournant religieux dans les études du développement}

2 En 2004, la Banque mondiale publiait un livre intitulé Mind, Heart, and Soul in the Fight Against Poverty dans lequel est soulignée la nécessité d'une collaboration entre organisations religieuses et organisations de développement pour lutter contre la pauvreté. Les élites chrétiennes et islamiques y sont considérées comme des partenaires incontournables pour la mise en œuvre de programmes dans des domaines aussi variés que la santé, l'éducation, l'environnement ou le maintien de la paix 
(Marshall \& Keough 2004). De même, le Rapport sur le Développement Humain 2007/2008 comprend un plaidoyer pour inclure des valeurs et des partenaires religieux dans la lutte contre le changement climatique ${ }^{1}$.

Cette attention explicite accordée à la religion ces dernières années dans les milieux de coopération pour le développement peut surprendre, sachant que la religion et le développement ont semblé de façon durable appartenir à des sphères distinctes. Avec le paradigme de la modernisation, devenu dominant dans les études et la pratique du développement à partir des années 1950 et 1960, la religion est supposée être hostile au changement, exerçant une force d'inertie destinée à disparaître avec la modernité et l'accès du plus grand nombre à l'instruction. A cette époque, la société civile encore balbutiante, n'a pas vraiment voix au chapitre.

4 Les choses commencent graduellement à changer à partir des années 1980. Avec les politiques d'ajustement structurel et le retrait de l'État de secteurs sociaux tels que l'éducation, la santé publique et les services sociaux, on assiste à une diversification des acteurs du développement (ONG, associations, etc.) qui tentent de combler le déficit d'État. Parallèlement à cela, les approches strictement macroéconomiques du développement sont remplacées (ou, dans bien des cas, complétées) par des perspectives plus holistes du « développement humain » (Sen 1997, 2000) qui vont audelà des approches purement économiques du développement pour inclure également des aspects sociaux, psychiques et culturels. Les démarches fondées sur le concept de 'livelihood' (Chambers \& Conway 1992 ; Carney 1998), qui mettent l'accent sur les capacités propres des individus et leurs stratégies de vie, gagnent particulièrement en influence. Elles s'associent aux politiques participatives, surtout parce qu'elles correspondent bien au paradigme néolibéral, axé sur la privatisation et la prise de leurs responsabilités par les citoyens (Kaag et al. 2004).

Toutefois, ces nouvelles approches se présentant comme holistes et centrées sur les personnes considérées plutôt comme des êtres humains dans leur entièreté que des acteurs économiques, conservent encore, au milieu des années 1980, leur caractère «laïque », mettant l'accent sur les aspects socio-économiques du développement humain. C'est seulement à la fin du siècle dernier que la religion et la culture viennent au coeur des analyses. Un exemple révélateur est la théorie du « choc des civilisations » (Huntington 1996) qui devient un prisme de compréhension des conflits géopolitiques. Cette idée selon laquelle la culture (y compris la religion) joue un rôle dans l'ère globale a inspiré d'autres observateurs. Contrairement à Huntington pour qui cette influence est négative, ceux-ci voient dans la religion une force plutôt positive et aidant le développement, comme montre le titre du rapport de la Banque Mondiale cité plus haut.

Dans le même temps, à rebours des visions simplistes et globalisantes, nombre de travaux mettent en avant le rôle crucial des institutions religieuses dans le fonctionnement des États africains contemporains et leur développement économique (Copans 1989 ; Fall 2011). Dans le cas du Sénégal, par exemple, l'accent a été mis sur l'islam comme une « contre-culture » politique populaire (Coulon 1983 ; Seck 2010), ou bien, au contraire, l'islam constitue un moyen dans les mains des élites politiques et religieuses pour maintenir et renforcer leur pouvoir (Gueye \& Seck 2011). En général, on reconnaît aux sphères religieuses un rôle non négligeable pour assurer une forme de protection sociale basée sur des principes de charité (Vuarin 1990). Dans le champ des études et de la pratique du développement, les religions se donnent alors à voir comme 
force de changement (Brenner 1993), à même de se constituer en ONG. Qu'elles aient pour objectif d'évangéliser tout en promouvant les économies locales (Yaro 1998), de participer à des campagnes de lutte contre la pandémie du Sida (Delaunay et al. 1998), les ONG confessionnelles sont perçues comme susceptibes d'être plus efficaces du fait de leur proximité culturelle supposée avec les groupes sociaux ciblés (Benthall 2007).

7 Au tournant des années 2000, les approches anthropologiques, liant religion et vie publique (Sounaye 2007) se multiplient. Elles mettent en évidence des engagements sociétaux forts (Kaag 2011), des formes de citoyenneté islamiques impliquant les militants religieux dans la vie de la cité (Gomez-Perez \& Leblanc 2007). Les cadets sociaux, jeunes et femmes, accèdent à des modes nouveaux d'expression publique (Masquelier 2009; Soares 2009). Dans le champ de ces récents travaux en anthropologie, le fait religieux est abordé de façon renouvelée dans ces liens au politique et à la chose publique. Mais le fait est que ces voix "anthropologiques " commencent tout juste à être prises en compte par les praticiens du développement ${ }^{2}$.

\section{Le renouveau religieux en action}

8 Parallèlement à cela, de nombreux changements s'opèrent à la faveur de ce que l'on qualifie souvent de "renouveau» ou de «réveil» religieux. En Afrique, si le fait religieux a toujours occupé une place importante dans la vie sociale des individus, on observe en effet depuis vingt ans des dynamiques nouvelles qui donnent au fait religieux une dimension publique. Aujourd'hui plus qu'hier, les militantismes religieux s'illustrent dans l'espace public. Le progrès technologique (Saint-Lary, Samson \& Amiotte-Suchet 2011), la globalisation et la mondialisation des discours religieux ne sont évidemment pas étrangers à tous ces changements. Les églises pentecôtistes et les mouvements réformistes islamiques déploient des moyens techniques importants pour s'approprier les médias de masse, ravivant du même coup les concurrences. On parle de réformismes religieux, qui prennent forme autant dans leur version islamique, que catholique ou protestante et conduisent les responsables religieux à diffuser des normes, rectifier des pratiques cultuelles, inculquer des valeurs sociales et morales.

Ce phénomène de "revival », qui s'observe à partir des années 1990, s'opère sous l'effet conjugué de plusieurs facteurs. En effet, côté musulman, dès les années $1970^{3}$, les contextes de la guerre du Kippour, du choc pétrolier et des grandes sécheresses subsahariennes se conjuguent pour renforcer les relations entre l'Afrique noire et le monde arabo-persan (Triaud 2007). La Révolution de la République islamique d'Iran (1979) donne de son côté à "cette nouvelle forme d'islam politique une visibilité internationale et suscite une certaine fascination dans les milieux musulmans » (Triaud 2007 : 10). Cette ouverture des pays arabes sur l'Afrique noire (Loimeier 2005 ; Bunza 2005; Miran 2006; Kaag 2007) et la coopération bilatérale qui en découle se matérialisent par une aide au développement conséquente (Cissé 1994). Du côté chrétien, les Eglises pentecôtistes ne sont pas en reste. Le modèle exemplaire de l'Eglise Universelle du Royaume de Dieu (EURD) en témoigne. En effet, l'EURD est bien connue pour sa capacité à s'emparer des médias et à mobiliser de manière exponentielle les fidèles (Corten, Dozon \& Oro 2003).

Ainsi, on assiste en Afrique au sud du Sahara à l'expansion d'un véritable «espace public religieux» (Holder 2009) où s'élaborent des stratégies de prosélytisme, certes, mais aussi des plans d'action pour améliorer le vivre ensemble. Les élites religieuses 
s'investissent aujourd'hui dans des combats aux visées à la fois cultuelles et sociopolitiques. La teneur des discours et de l'action publics en témoignent. On le voit dans la plupart des contributions de ce numéro du Bulletin de l'APAD, les organisations religieuses participent à la construction d'établissements publics de santé et d'éducation, de même qu'elles instaurent, dans les interstices de leur système, des formes de solidarité pour faire face aux fléaux ou pallier des crises conjoncturelles.

\section{L'implication des élites religieuses dans l'arène du développement}

11 La nouvelle visibilité des idées et des acteurs religieux dans le "développement» apparaît donc comme un phénomène important à analyser. Un des partis pris de ce numéro est de considérer le lien entre religion et développement au-delà des seules organisations religieuses et ONG fondées sur la foi (Bornstein 2003; Hofer 2003). Il s'agit en effet d'aborder le sujet en adoptant une perspective historique et en considérant pleinement le rôle des élites religieuses, qu'elles soient structurées sur des organisations ayant une valeur juridique ou sur des groupes informels. De plus, les contributions de ce numéro, loin de se limiter aux dynamiques locales, intègrent les dimensions nationales et transnationales du phénomène. Les articles montrent par exemple que les élites religieuses peuvent être des intermédiaires entre des organisations internationales et locales, comme l'association sénégalaise islamique Jamra (Gomez-Perez dans ce numéro). Leurs activités sont financées tant par les organisations internationales, que par les pays du Golfe, ou de façon interne, par les fidèles eux-mêmes.

12 En outre, le caractère polysémique du terme "élites " ne doit pas tromper car cette catégorie comprend autant de détenteurs des savoirs religieux, dotés parfois de diplômes en théologie, que les prédicateurs formés plutôt sur le tas, mais non moins actifs dans la vie de la cité. Les élites religieuses ont précisément pour caractéristiques de bénéficier d'un pouvoir d'influence auprès des croyants. Qu'elles tiennent cette légitimité de leur savoir et/ou de leur capital relationnel et charismatique, le résultat est là : elles agissent dans l'espace public et y transmettent des messages à forte portée sociétale. Les études dans ce numéro se portent sur les positions, les stratégies et les perceptions de ces élites religieuses engagées dans le « développement » d'une façon ou d'une autre, et cela dans différents contextes ouest-africains (Niger, Burkina Faso, Sénégal, et Nigeria), et au cours de différentes périodes (depuis les temps précoloniaux jusqu'à récemment).

13 Ainsi, Nasir Mohammed Baba analyse le rôle des ulama (les savants musulmans) dans la promotion de l'éducation islamique au Nigeria septentrional depuis la période précoloniale. Il décrit le schéma historique complexe de coopération, de compétition et d'évitement qui caractérise la relation entre les ulama et l'état dans le domaine de l'éducation. Il conclut que l'État reconnaît actuellement l'importance d'une éducation de base de bonne qualité pour tous, mais que celui-ci n'est pas en mesure de réguler la participation des élites et des groupes islamiques dans l'offre d'éducation. Baba suggère que l'impression grandissante d'isolement des écoles islamiques a conduit les élites religieuses à renforcer leur contrôle sur le contenu des programmes au point de freiner le développement de l'éducation et l'unité nationale. 

initiatives du développement islamique au Niger au cours de la dernière décennie. Il observe en particulier comment une voie de développement à la manière islamique a émergé dans la sphère publique et a mobilisé une diversité d'acteurs musulmans. En comparant les points de vue et les activités de l'ONG islamique internationale, AMA (Agence des Musulmans d'Afrique) et des entrepreneurs islamiques locaux du développement, Abdoulaye Sounaye analyse ce que faire du développement à la manière islamique veut dire, en termes de conséquences sur leurs relations avec les " groupes cibles ".

Pour sa part, Katrin Langewiesche traite de la question du dialogue interreligieux au Burkina Faso, un thème curieusement peu abordé dans le champ des sciences sociales. Elle analyse les partenariats, qui, dans le domaine de la santé, se sont constitués entre des organisations religieuses et l'État dans le courant des dix dernières années. Elle montre ainsi que les élites religieuses sont de plus en plus impliquées dans la transmission de messages concernant la santé publique. De plus, dans le contexte de pluralité qui caractérise le Burkina Faso, le dialogue interreligieux apparaît comme une stratégie mobilisée par les élites religieuses pour occuper des positions de force dans l'arène du développement et être favorablement perçues par l'État et des donateurs internationaux.

16 Muriel Gomez-Perez considère, de façon plus spécifique, l'expérience de deux ONG islamiques au Sénégal dans leur lutte contre le VIH/Sida. Elle montre comment ces organisations prennent place dans un contexte de retrait de l'État et de forte mobilisation dans la lutte contre le Sida. Elles répondent à une demande internationale et parviennent à faire entrer des leaders religieux dans la bataille contre le Sida. Parallèlement à cela, elles développent leurs propres approches inspirées et légitimées par le Coran et les Hadiths, quitte à adopter une posture critique vis-à-vis de l'État et de la communauté internationale. Quelle que soit la variété des acteurs et des contextes traités dans ce numéro, certains thèmes communs se profilent.

\section{Des liens de collaboration et de concurrence}

Les acteurs religieux et du "développement", comme l'État, les organisations internationales ou les ONG laïques, entretiennent des liens qui vont de la franche collaboration à la rivalité plus ou moins explicite.

Parfois, le religieux peut se poser en véritable concurrent critiquant ouvertement les actions des pouvoirs publics et des ONG laïques jugées immorales ou en contradiction avec les préceptes religieux. Les conflits de normes mènent alors à de véritables bras de fer entre institutions religieuses et laïques dans l'arène du développement. Comme le montre Muriel Gomez-Perez, les élites islamiques perçoivent les distributions massives de préservatifs comme une incitation à la débauche et rejettent fermement, à l'instar du Pape, les institutions qui se prêtent à ce type d'action.

Néanmoins, les arènes du développement donnent à voir des rapports, entre les acteurs religieux et politiques, fondés sur l'interdépendance et la collaboration, que celles-ci soient formelles ou informelles, dans le discours public ou dans l'action. L'influence des élites religieuses est largement mobilisée pour légitimer des programmes de sensibilisation. Ceci s'observe notamment dans le domaine de la lutte contre la 
pandémie du Sida (contributions de Katrin Langewiesche et Muriel Gomez-Perez dans ce numéro). Ainsi, nombre d'acteurs religieux, investis dans un combat à portée panafricaine et mondiale, considèrent l'importance des idéaux du développement : la planification familiale, la vaccination, la lutte contre la pauvreté, sont bel et bien des enjeux qui concernent musulmans et chrétiens, d'autant plus que, depuis quelques années, la conjoncture économique les mobilise.Les organisations confessionnelles travaillent à démontrer leur légitimité dans les milieux du développement. Ainsi au Burkina Faso, elles mettent en avant le dialogue interreligieux, conscientes qu'il constitue pour les organisations internationales une configuration "religieusement correct ». En tout état de cause, il ressort que du côté des organisations internationales, beaucoup ont compris que leur idéal participatif ne peut être accompli que grâce à l'aide des personnages charismatiques, écoutés et influents, parmi lesquels on compte bel et bien les leaders religieux. On assiste donc à une présence de plus en plus importante de courtiers religieux du développement, qui s'érigent en traducteurs des textes canoniques, pour montrer aux populations ciblées par ces actions leur bien fondé sur le plan religieux. Comme le montre Abdoulaye Sounaye dans ce numéro, ces acteurs cumulent un double registre de compétence fondé sur leur connaissance de la langue officielle et des savoirs islamiques qui leur permettent d'argumenter théologiquement leur propos. En citant les Hadith appropriés, ils sensibilisent la population en faveur des campagnes de vaccination.

En outre, le religieux étant souvent pluriel, la coopération des uns avec les pouvoirs publics fait parfois le mécontentement des autres qui les accusent d'être corrompus. Ceci pose donc la question des segmentations religieuses face à une gestion conjointe avec les pouvoirs publics des problèmes sociétaux. Dans le jeu des concurrences au sein de la sphère islamique, certains acteurs ont pour mission de contrer le discours antioccidental de leurs coreligionnaires à leurs yeux contreproductif en matière de développement. Au sein d'une même confession, les lectures des textes religieux peuvent varier et conduire à des visions différentes des actions de sensibilisation (Muriel Gomez-Perez).

21 De manière plus globale, les discours religieux s'appuient largement sur une rhétorique du salut incitant les croyants à œuvrer ici-bas en pensant à l'Au-delà. Ils construisent une économie morale du salut au sein de laquelle les actions publiques de développement prennent corps.

\section{Le religieux dans la construction de l'État-Providence}

Ces dynamiques s'ancrent dans un contexte de crise, financière et économique, qui a conduit en vingt ans le continent vers une réelle paupérisation des populations tant urbaines que rurales. Les espoirs qu'avaient engendrés les processus de démocratisation amorcés dans les années 1990, font aujourd'hui place à une déception généralisée. Le constat n'est pas très reluisant : défaillance et privatisation des États, crises céréalières à répétitions (2004-2005), révoltes contre la vie chère, coupures d'électricité, dysfonctionnements des systèmes de santé, urbanisation sauvage. Face à ce que d'aucuns qualifient de crise de la grande famille, les solidarités communautaires étant mises à mal, les réseaux religieux de solidarité viennent combler cette précarité. Ils se substituent à l'État, dans ses fonctions sociales et protectrices et s'emparent des politiques publiques liées au genre, à la lutte contre la pauvreté, à l'éducation ou à la 
santé. Il faut dire que de plus en plus, le budget des organisations religieuses, chrétiennes et musulmanes, le leur permette.

En effet, pendant longtemps, ces réseaux se sont essentiellement nourris de l'extérieur : du côté islamique, les pétrodollars ont largement contribué (et contribuent encore aujourd'hui) à financer des actions de développement et à nourrir nombre d'ONG. Côté chrétien, les financements États-uniens des réseaux pentecôtistes en Afrique leur ont valu d'être appelés «américains». Dans leur version plus récente et plus fondamentaliste, les néopentecôtistes comme l'Eglise Universelle du Royaume de Dieu puisent largement leur économie au Brésil, tous ces financements ayant ainsi conduit à structurer des hiérarchies autour d'une forte dépendance avec l'extérieur.

Aujourd'hui, au-delà de ces financements extérieurs, les leaders religieux locaux ont su mobiliser leurs troupes et rétablir ainsi une relative autonomie économique. Désormais, la sphère religieuse bénéficie d'une assise économique alimentée par les fidèles eux-mêmes qui cotisent et consacrent parfois des parts importantes de leur budget aux institutions religieuses. Ce pouvoir économique de la sphère religieuse ne constitue pas une nouvelle donne des sociétés africaines, mais aujourd'hui, prend des formes et dimensions particulières. Qu'il s'agisse de la zakat (aumône légale au sens islamique du terme), du mécénat des grands entrepreneurs (Cissé 2009), des dons ostentatoires aux pasteurs (Furtado, Laurent \& Plaideau 2009), des bénédictions, véritable "économie de la prière " (Soares 1996 ; Last 1988), avec dons de numéraires effectués lors de célébrations comme le Mouloud (Saint-Lary 2006), qu'il s'agisse des cotisations aux associations ou du marketing religieux (Soares 2007) (pacotilles, affiches, cassettes, opuscules), le marché du religieux s'intercale entre économie de marché et économie morale (Triaud \& Villalòn 2009). Il permet véritablement à une sphère religieuse d'exister et de prendre place dans l'arène du développement, avec une relative marge de manœuvre sur ses objectifs. Elle n'est ni exclusivement financée par des institutions laïques qui ont besoin de la caution religieuse, ni exclusivement par la hiérarchie religieuse étrangère, elle s'autofinance en partie, et donc s'autonomise dans le champ du développement. Les élites religieuses, de ce fait, participent pleinement, avec leurs moyens propres, du fonctionnement des États africains.

Tous ces phénomènes, qui laissent entrevoir l'émergence d'un État providence religieux - ou de ce que Maria Das Dores Campos Machado (2003) qualifie d' " organisation providence " - posent à nouveaux frais la question de la laïcité. En effet, cette participation des sphères religieuses aux politiques publiques montre combien la sécularisation est loin d'entraîner comme en Europe, un processus de perte d'influence sociale de la religion dans les sociétés modernes (Willaime 2006). L'existence d'un « religieux postmoderne » ou pour reprendre l'expression d'Hassane Souley (2009), d'un islam postmoderne, n'empêche pas que les États concernés soient laïques, avec une conception singulière de ce principe. Les trois fondements de la laïcité, à savoir l'égalité de traitement entre les religions, la liberté de culte et la séparation institutionnelle entre le religieux et le politique (Baubérot 2007), s'articulent dans les contextes ouest-africains de façon particulière. Aussi, considérer le rôle des élites religieuses dans les arènes du développement, c'est envisager l'existence de laïcités à l'africaine ${ }^{4}$ où le religieux participe à la construction et au fonctionnement des États africains, sans nécessairement les conquérir (quoi que cela ne soit définitivement pas exclu). Ce que ces élites religieuses cherchent à conquérir est moins 
l'État au sens régalien du terme (justice, police etc.) que l'État-providence. La dimension de cette conquête demeure plus sociale que politicienne.

\section{BIBLIOGRAPHIE}

BAUBÉROT, J.,2007. Histoire de la laïcité en France, Paris, PUF (Que sais-je ?), $4^{\mathrm{e}}$ édition.

BENTHALL, J., 2007. Islamic Charities in Southern Mali Today. Islam et sociétés au sud du Sahara 1, 2007, pp. 165-174.

BORNSTEIN, E., 2003. The Spirit of Development: Protestant NGOs, Morality, and Economics in Zimbabwe. London and New York: Routledge.

BRENNER, L., 1993. Muslim Identity and Social Change in Sub-Saharan Africa. London: Hurst.

BRENNER, L., 2001. Controlling Knowledge. Religion, power and schooling in a West-African Muslim society. Bloomington: Indiana University Press.

BUNZA, MUKHTAR UMAR, 2005. « The Iranian model of political Islamic movement in Nigeria (1979-2002), in Gomez-Perez (dir.), L'islam politique au Sud du Sahara. Identités, discours et enjeux, Paris, Karthala : 227-241.

CARNEY, D. (ed.) 1998. Sustainable Rural Livelihoods: What Contribution Can We Make? London, DFID.

CHAMBERS, R. \& G. R. CONWAY, 1992. Sustainable Rural Livelihoods: Practical Concepts for the $21^{\text {st }}$ Century, Sussex, IDS.

CISSÉ, I., 2009. « Le wahhabisme au Burkina Faso : dynamique interne d'un mouvement islamique réformiste ", Cahiers du CERLESHS, tome XXIV, n³3:1-33.

CISSÉ, I., 1994. Islam et État au Burkina Faso : de 1960 à 1990, Thèse (Triaud dir.), Aix-en-Provence, Université de Provence, 2 Vol.

COOPER, B., 2006. Evangelical Christians in the Muslim Sahel. Bloomington: Indiana University Press. COPANS, J., 1989. Les Marabouts de l'arachide : la confrérie mouride et les paysans du Sénégal, Paris : le Sycomore, « Les Hommes et leurs signes".

COULON, C., 1983. Les musulmans et le pouvoir en Afrique noire, Paris, Karthala.

CORTEN, A., DOZON, J-P. \& ORO, A. P., 2003. Les nouveaux conquérants de la foi. L'Eglise Universelle du Royaume de Dieu (Brésil), Paris, Karthala.

DELAUNAY, KARINE, 1998. « Des ONG et des associations: concurrences et dépendances sur un "marché du Sida" émergent. Cas ivoirien et sénégalais ", inDeler, J-P., Fauré, Y.A., Piveteau, A. et Roca, P.J. (dir.), ONG et développement. Société, économie, politique, Karthala : 115-141.

DOZON, J-P., 2009. « Remémoration coloniale et actualisation politique dans la confrérie mouride », L'Islam, nouvel espace public en Afrique, Paris, Karthala : 225-236.

FALL, A.S., 2011. « Les usages des liens confrériques dans l'économie sénégalaise. », in Kaag, M. (dir), Islam et engagements au Sénégal. Leiden : African Studies Centre. 
FURTADO, C., LAURENT, P-J \& PLAIDEAU, C., 2009. « L’Eglise Universelle du Royaume de Dieu du Cap-Vert. Croissance urbaine, pauvreté et mouvement néo-pentecôtiste ", Bulletin de l'APAD, $\mathrm{n}^{\circ}$ $29-30: 9-25$.

GUEYE, C, et A. SECK, 2011. «Islam et politique au Sénégal : logique d'articulation et de coproduction » in Kaag, M. (dir), 2011, Islam et engagements au Sénégal. Leiden : African Studies Centre.

GOMEZ-PEREZ, M., 2005. L'islam politique au Sud du Sahara. Identités, discours et enjeux, Paris, Karthala.

GOMEZ-PEREZ, M. \& LEBLANC, M-N, 2007. « Jeunes musulmans et citoyenneté culturelle : retour sur des expériences de recherche en Afrique de l'Ouest francophone », Sociologie et sociétés, vol 39 (2) : 39-59.

HASSANE, S., 2009. "Société civile islamique et nouveaux espaces publics au Niger. Esquisse sur l'islam postmoderne et les pratiques religieuses "globales" en Afrique, in Holder (dir.), L'Islam, nouvel espace public en Afrique, Paris, Karthala : 101-125.

HODGKIN, E., 1998. « Islamism and Islamic Research in Africa », in Kane et Triaud (éds), Islam et islamismes au sud du Sahara, Paris, Karthala : 197-262.

HOLDER, G., 2009. L'Islam, nouvel espace public en Afrique, Paris, Karthala.

HOFER, K., 2003. The Role of Evangelical NGOs in International Development: A Comparative Case Study of Kenya and Uganda, Afrika Spectrum 38(3): 373-398.

HUNTINGTON, S., 1996.The Clash of Civilizations and the Remaking of World Order. New York: Simon \& Schuster.

KAAG, M. et al.2004. 'Ways forward in livelihood research.' In: Kalb, D., W. Pansters \& H. Siebers, Globalization \& Development. Themes and Concepts in Current Research, Dordrecht, Boston \& London: Kluwer Academic Publishers.

KAAG, M., 2007. 'Aid, Umma and Politics: Transnational Islamic NGOs in Chad', in R. Otayek \& B. Soares (eds), Muslim Politics in Africa, New York: Palgrave Macmillan, pp. 85-102.

KAAG, M. (éd), 2011. Islam et engagements au Sénégal. Leiden : African Studies Centre.

KRZNARIC, R., 2007. For God's Sake, Do something! How Religions Can find Unexpected Unity Around Climate Change. Human Development Report 2007/2008 Occasional Paper. Human Development Report Office.

LAST, M., 1988. "Charisma and Medicine in Northern Nigeria", In : Cruise O’Brien, D. B. et C. Coulon eds., Charisma and Brotherhood in African Islam. Oxford, Clarendon Press : 183-204.

LOIMEIER, R., 2005. « De la dynamique locale des réformismes musulmans. Etudes biographiques (Sénégal, Nigeria, Afrique de l'Est) », in Gomez-Perez (dir.) L'islam politique au Sud du Sahara. Identités, discours et enjeux, Paris, Karthala.

MACHADO, M., 2003. “L'IURD : une “organisateur providence”, in Corten, Dozon et Oro (dir.), Les nouveaux conquérants de la foi. L'Eglise Universelle du Royaume de Dieu (Brésil), Paris, Karthala ; 245-256.

MARSHALL, K. \& KEOUGH, L., 2004. Mind, Heart, and Soul in the Fight against Poverty.Washington DC: World Bank.

MASQUELIER, A., 2009. Women and Islamic Revival in a West African Town, Bloomington: Indiana University Press. 
MIRAN, M., 2006, Islam, histoire et modernité en Côte d'Ivoire, Paris, Karthala.

OLIVIER DE SARDAN, J-P., 2000. « Dramatique déliquescence des États en Afrique », Le Monde diplomatique, février-2000.

OTAYEK, R. 1993. « Une relecture islamique du projet révolutionnaire de Thomas Sankara », in : Bayart, J.-F. (éd.), Religion et modernité en Afrique Noire : 101-127.

SAINT-LARY, M., 2006. Les chefs peuls du Yatenga à l'épreuve du changement (Burkina Faso), Thèse, EHESS, Marseille.

SAINT-LARY, M, SAMSON, F. \& AMIOTTE-SUCHET, L. 2011. Les outils d'un islam en mutation. Réislamisation et moralisation au Sud du Sahara, Ethnographiques.org.

SECK, A., 2010. La question musulmane au Sénégal. Essai d'anthropologie d'une nouvelle modernité, Paris, Karthala.

SEN, A., 1997. Editorial: Human Capital and Human Capability, World Development, 25(12): 1959-1961.

SEN, A., 2000. Human Development and Economic Sustainability, World Development, 28 (12): 2029-2049.

SOARES, B. F., 1996. "The prayer economy in a Malian town", Paris, Cahiers d'Etudes Africaines, 36 (4): 739-753.

SOARES, B. F., 2005. Islam and the Prayer Economy. History and Authority in a Malian Town, Edinburgh: Edinburgh University Press.

SOARES, B. F., 2007. "Saint and Sufi in contemporary Mali", In M.van Bruinessen and J.D. Howell (eds.), Sufism and the 'modern' in Islam, London I.B. Tauris : 76-91.

SOARES, B.F., 2009. "An Islamic social movement in Contemporary West-Africa: NASFAT of Nigeria”, In: S. Ellis and I.van Kessel, Movers and Shakers: Social Movements in Africa, Leiden: Brill: 178-196.

SOUNAYE, A, 2007. “Instrumentalizing the Qur'an in Niger's Public Life”, Journal for Islamic Studies, vol.27: 211-239.

TER HAAR, G. \& S. ELLIS, 2006. The Role of Religion in Development: Towards a New Relationship between the European Union and Africa. The European Journal of Development Research, vol. 18, no. 3, September 2006, pp. 351-367.

TRIAUD, J.-L., 2007. «L'islam au sud du Sahara : pistes de réflexion », In Souley, Moyet, Seck et Zakari, Islam sociétés et politique en Afrique Subsaharienne. Les exemples du Sénégal, du Niger et du Nigéria, Paris, Les Indes savantes.

TRIAUD, J.-L., \& VILLALON, L., 2009. « L'islam subsaharien entre économie morale et économie de marché : contraintes du local et ressources du global », Afrique contemporaine (3), n 231 : 23-42. UNITED NATIONS DEVELOPMENT PROGRAMME, 2007. Human Development Report 2007/2008 Fighting Climate Change. Human solidarity in a divided world. New York: Palgrave Macmillan. VUARIN, ROBERT, 1990. «L'enjeu de la misère pour l'islam sénégalais », Tiers-Monde, tome 31, $n^{\circ} 123: 601-621$.

WILLAIME, J-P., 2006. « La sécularisation : une exception européenne ? Retour sur un concept et sa discussion en sociologie des religions », Revue Française de Sociologie, 47-4: 755-783. 
YARO, G.J., 1998. « De l'évangélisation au développement local. Spécificité des ONG d'inspiration protestante au Burkina Faso », in Deler, Fauré, Piveteau et Roca (dir.), ONG et développement. Société, économie, politique, Paris, Karthala : 143-155.

\section{NOTES}

1. Voir en particulier l'un des textes accompagnant le rapport, intitulé «Pour l'amour de Dieu, faites quelque chose! Comment les religions peuvent trouver une unité inattendue autour du changement climatique » (Krznaric 2007).

2. Il convient de remarquer que la "découverte" de la religion par les théoriciens et les praticiens du développement semble impliquer une appréciation (renouvelée) de l'anthropologie en tant que discipline dans ces cercles.

3. Jean-Louis Triaud indique deux autres périodes importantes dans l'histoire de l'expansion de l'islam en Afrique subsaharienne, à savoir le XVIIIème siècle, période des jihad peuls, et l'époque coloniale.

4. On se réfère ici au colloque qui s'est tenu récemment sur le thème, "L'Afrique des laïcités ", Bamako, Janvier 2010. 